\title{
Transduplication resulted in the incorporation of two protein-coding sequences into the Turmoil-I transposable element of C. elegans
}

Noa Sela ${ }^{1}$, Adi Stern ${ }^{2}$, Wojciech Makalowski ${ }^{3}$, Tal Pupko ${ }^{2}$ and Gil Ast*1

Address: ${ }^{1}$ Department of Human Molecular Genetics and Biochemistry, Sackler Faculty of Medicine, Tel Aviv University, Tel Aviv 69978, Israel, ${ }^{2}$ Department of Cell Research and Immunology, George S. Wise Faculty of Life Sciences, Tel Aviv University, Tel Aviv 69978, Israel and ${ }^{3}$ Institute of Bioinformatics, Faculty of Medicine, University of Muenster, Muenster D-48149, Germany

Email: Noa Sela - noasela@post.tau.ac.il; Adi Stern - sternadi@post.tau.ac.il; Wojciech Makalowski - wojmak@uni-muenster.de; Tal Pupko - talp@tauex.tau.ac.il; Gil Ast* - gilast@post.tau.ac.il

* Corresponding author

Published: 8 October 2008

Biology Direct 2008, 3:4I doi:10.1I86/I745-6/50-3-4I

This article is available from: http://www.biology-direct.com/content/3/l/4I

(C) 2008 Sela et al; licensee BioMed Central Ltd.

This is an Open Access article distributed under the terms of the Creative Commons Attribution License (http://creativecommons.org/licenses/by/2.0), which permits unrestricted use, distribution, and reproduction in any medium, provided the original work is properly cited.

\begin{abstract}
: Transposable elements may acquire unrelated gene fragments into their sequences in a process called transduplication. Transduplication of protein-coding genes is common in plants, but is unknown of in animals. Here, we report that the Turmoil-I transposable element in C. elegans has incorporated two protein-coding sequences into its inverted terminal repeat (ITR) sequences. The ITRs of Turmoil-I contain a conserved RNA recognition motif (RRM) that originated from the rsp2 gene and a fragment from the protein-coding region of the $\mathrm{cpg}-3$ gene. We further report that an open reading frame specific to $C$. elegans may have been created as a result of a Turmoil- $I$ insertion. Mutations at the $5^{\prime}$ splice site of this open reading frame may have reactivated the transduplicated RRM motif.
\end{abstract}

Reviewers: This article was reviewed by Dan Graur and William Martin. For the full reviews, please go to the Reviewers' Reports section.

\section{Findings}

The possible contribution of transposable elements to the proteome has been discussed in several publications [1-7] and has provoked much debate [8]. Many mechanisms are known to increase the protein-domain repertoire, e.g., domain duplication, substitution mutations, insertions, deletions, and domain rearrangements [9]. In metazoans, a transposable element may result in transduction, in which a DNA segment downstream of transposable elements is mobilized as part of an aberrant transposition. This may result in gene duplication or exon shuffling, subsequently enriching the protein repertoire [10-13]. How- ever, in the process of transduction, the transposable element does not acquire gene fragments as part of its sequence.

In plants, on the other hand, thousands of transposable elements contain duplicated gene fragments, captured in a process termed transduplication. Transduplication is a potentially rich source of novel coding sequences within rice and Arabidopsis thaliana [14-16]. Recently, transduplications of small nucleolar (sno) RNA by retroposon-like non-LTR transposable elements were found in the C. elegans [17] and platypus genomes [18]. 
The Harbinger superfamily of "cut-and-paste" DNA transposons was discovered through in silico studies [19]. This superfamily is characterized by Harbinger-specific transposases that are distantly related to the transposases encoded by the IS5-like group of bacterial transposons, such as IS5, IS112, and ISL2. Harbinger transposons are not as widespread as the eukaryotic $h A T$ and mariner $/ T c 1$ transposons; they are found in plants and nematodes [1923] but not in mammals. Usually, Harbinger transposons are flanked by 3-bp target site duplications and 25- to 50bp inverted terminal repeats (ITRs).

Turmoil-1 is a 5,024-bp long DNA transposon with 760bp long ITRs and a Harbinger-specific transposase (Figure 1A). These ITRs are unique to Turmoil-1 and are not found in other members of the Harbinger superfamily of DNA transposons [24]. One complete copy of the Turmoil-1 was found on chromosome II of C. elegans; eight Turmoil-1 fragments exist in the genome (for detailed information, see Table 1). An analysis of C. elegans transposable elements $[25,26]$ revealed that a 205 -bp ITR sequence within Turmoil- 1 is highly similar to a region of two exons separated by an intron of the rsp-2 gene (see pairwise alignment using bl2seq [27] Figure 1B). These two exons encode the RNA Recognition Motif (RRM), which is found in many eukaryal and bacterial proteins. Specifically, the type of RRM domain present in the rsp-2 (called RRM1) gene is highly conserved evolutionarily [28]. The high similarity between the ITR sequence and the fragment of the rsp-2 gene implies that one originated from the other. The antiquity of this domain and a phyloge- netic analysis (Figure 2) indicate that Turmoil-1 has recently acquired a portion of the $r s p-2$ gene sequence into its ITR. Tree reconstruction was performed with the PhyML program version 2.4.5 [29] using among-site rate variation with four discrete rate categories, and the JTT model [30] of sequence evolution.

A comparative analysis of the $r s p-2$ gene and the 205-bp region of the gene found in the ITR sequence, revealed that the Turmoil-1 sequence has accumulated several point mutations within the 5 ' splice site that make it non-functional, whereas the 3 ' splice site of the intron remains intact (the mutations in the $5^{\prime}$ splice site region are marked in red in Figure 1B). Since the RRM domain within the Turmoil-1 DNA transposon is not under purifying selection to maintain the reading frame or the functionality of the splice sites, these mutations are not unexpected.

Within the same ITR domain of Turmoil-1, and very close to the site of insertion of the RRM domain of $r s p$-2 gene, there is evidence of another "DNA kidnapping" event. A 131-bp fragment from the coding region of $C$. elegans gene $c p g-3$, which is unique to nematodes, was inserted into the ITR (Figure 1C). No sequences homologous to Turmoil-1 flank the $c p g-3$ gene. Thus, similar to the rsp-2 case, a gene fragment from the cpg-3 most likely was acquired by Turmoil-1, and not vice-versa. As the gene fragments are present on both sides of the ITR, capture may have occurred through non-homologous recombination.

Table I: Turmoil-I elements within the $C$. elegans genome

\begin{tabular}{|c|c|c|c|c|c|c|c|}
\hline \# & $\operatorname{Chr}(*)$ & Start $(* *)$ & End $(* *)$ & orientation & Containing RRM domain (***) & Containing cpg-3 fragment & Length of the element $(* * * *)$ \\
\hline I & 1 & 216923 & 217647 & - & $+(I-827)$ & + & PL \\
\hline 2 & 1 & 5979173 & 5980185 & + & $+(I-1177)$ & + & PL \\
\hline 3 & 1 & 5980183 & 5982782 & - & $+(I-277 I)$ & + & PL \\
\hline 4 & 1 & 7245338 & 7245520 & + & $-(|25-3| 7)$ & + & PL \\
\hline 5 & 1 & 13973017 & 13973196 & + & $-(469 \mid-4882)$ & + & PL \\
\hline 6 & II & 3351574 & 3356982 & - & $+(I-5024)$ & + & $\mathrm{FL}$ \\
\hline 7 & II & 10883165 & 10884063 & - & $-(519-1090)$ & - & PL \\
\hline 8 & II & | |45262| & II452867 & - & $+(323-568)$ & - & PL \\
\hline 9 & IV & 2900974 & 2901153 & + & $-(125-3 \mid 6)$ & + & PL \\
\hline
\end{tabular}

(*) chromosome number

(**) Start and End according to the coordinates of $C$. elegans genome version ce6 (May 2008).

$(* * *)$ in brackets the start and end relative to the full sequence of the Turmoil-I found on chromosome II 335I574-3356982 (\#6).

(****) PL - partial length of Turmoil- I/FL - full length of Turmoil-I. 


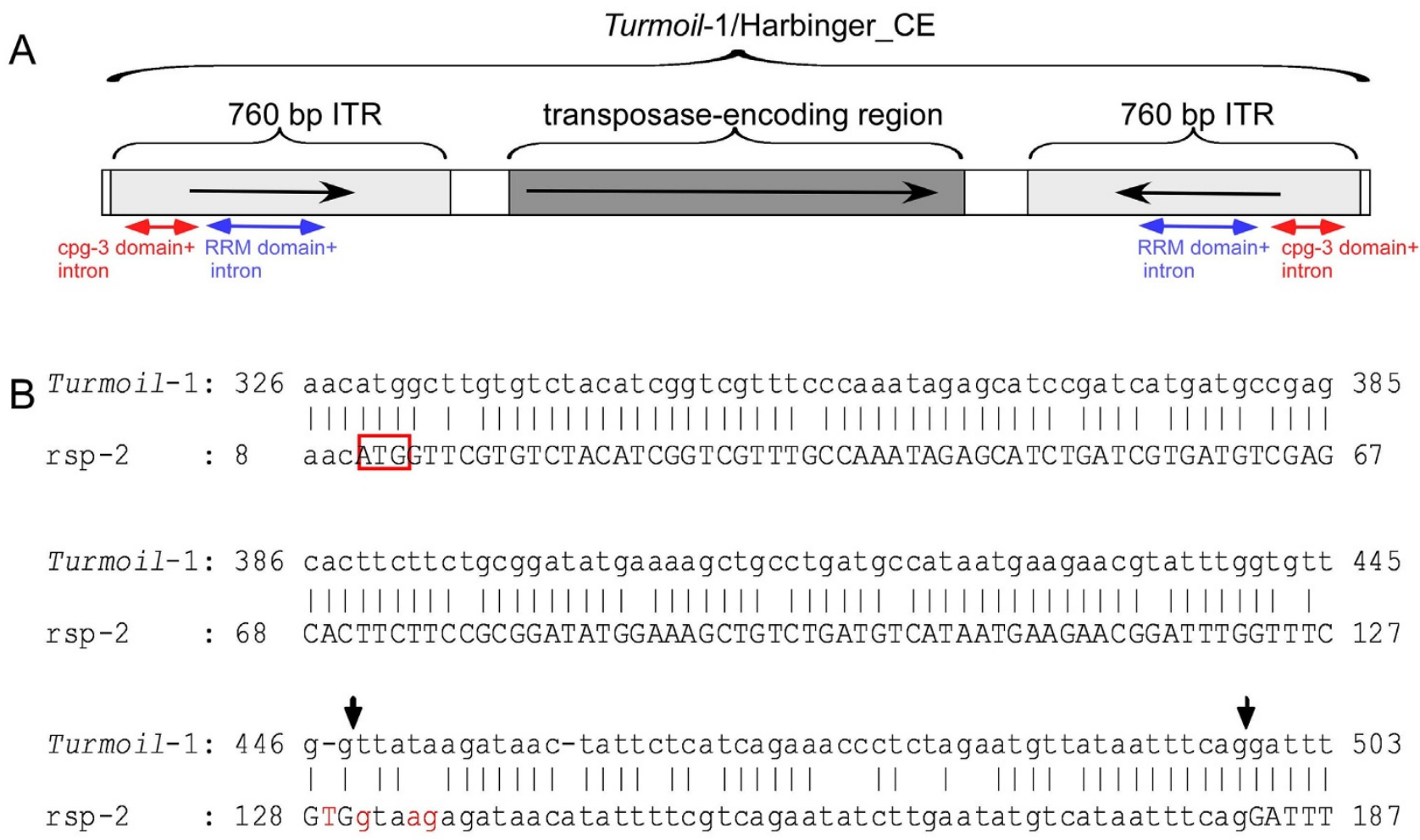

Turmoil-1: 504 tcaagatcagagcgatgctgacgac 528

|||||| ||| ||||||| || |||

rsp-2 : 188 TCAAGACCAGCGCGATGCCGATGAC 212

C Turmoil-1: 149 ctcactcttcaacagtccgagatgctcaacgcgtgtgctcaatatgctcaaacgggatca 208

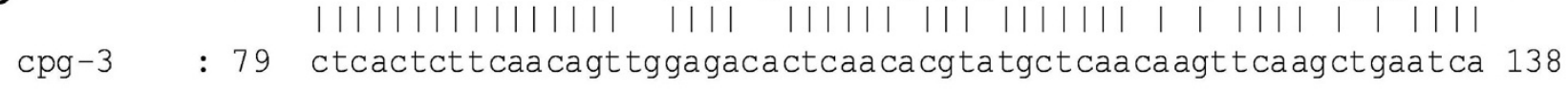

Turmoil-1: 209 cagaagctcactcgagaagctaactacgttatcacggaggttag 252

$\begin{array}{ccc}\text { Cpg-3 } & \text { : } 139 \text { cagaagctcattcaccaagctaacttcgtcatcactgaggttag } 182\end{array}$

\section{Figure I}

Evidence for two transduplication events within the ITR sequence of the Turmoil-I transposon. (A) A schematic illustration of the full sequence of Turmoil-I on chromosome II (see table I, copy number 6) and the structure and sites of inclusion of fragments of the rsp-2 and cpg-3 genes within the ITR sequence (B) Pairwise alignment (using bl2seq [27]) between the Turmoil-I sequence and the rsp-2 sequence. The start codon, which is also the first amino acid of the RRMI domain in the $r s p-2$ gene, is boxed in red. The intron sequence is indicated by lower-case letters; the protein-coding region is indicated by upper-case letters. The positions of the 5' and 3' splice sites (5'ss and 3'ss, respectively) are marked with arrows. The nucleotides in the 5 ' splice site of the rsp-2 gene that were mutated in the sequence of the transposable element, thereby abolishing the original 5' splice site, are shown in red. (C) Pairwise alignment between Turmoil-I and the cpg-3 sequence. 


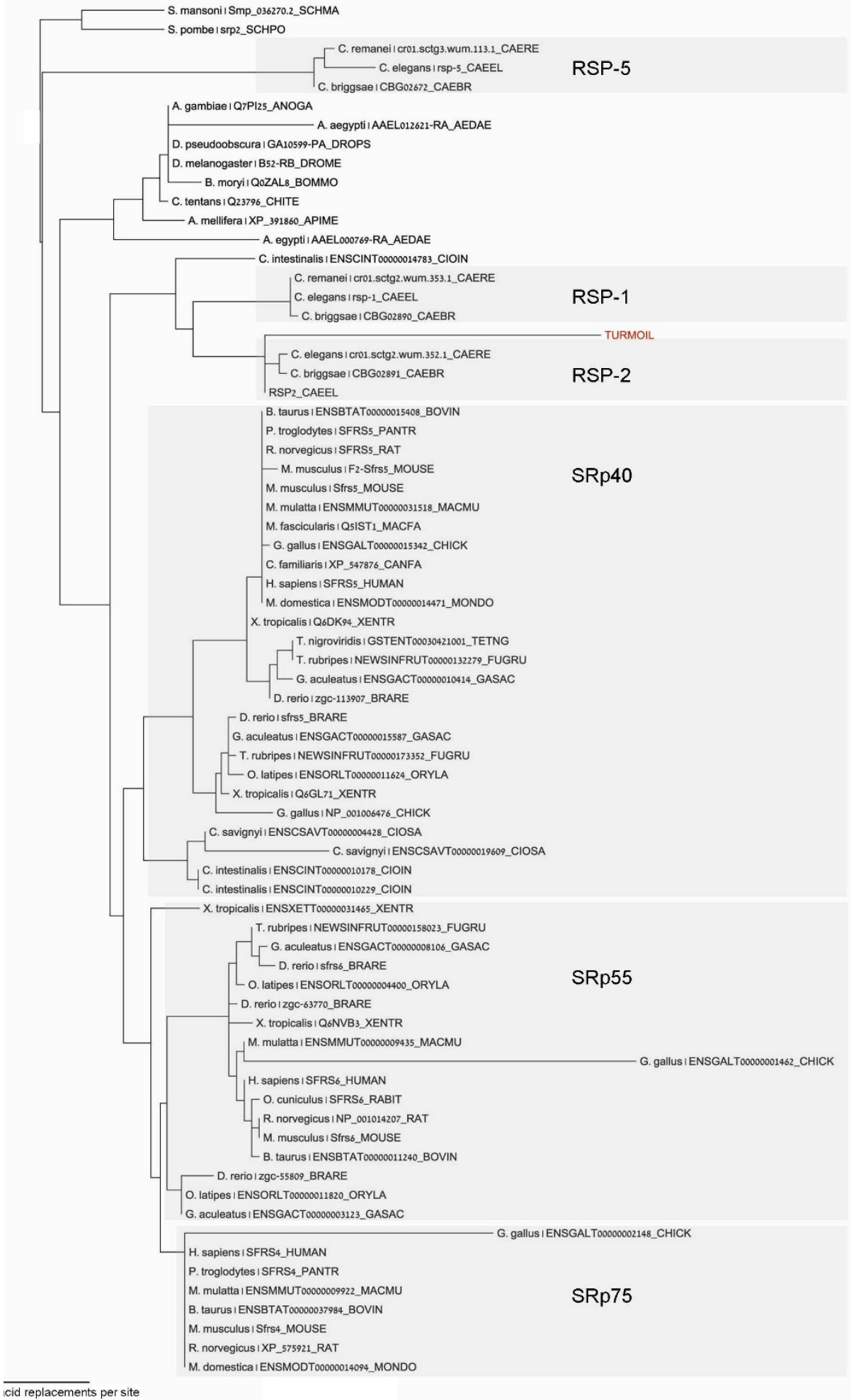

Figure 2

Maximum likelihood tree of the RRM domain. The RRM domain sequence, which is part of the ITR of Turmoil-I, is indicated in red. The tree shows that the RRM domain within Turmoil-I is derived from the ancestral RRM domain rather than vice versa. 
Harbinger/Turmoil-1

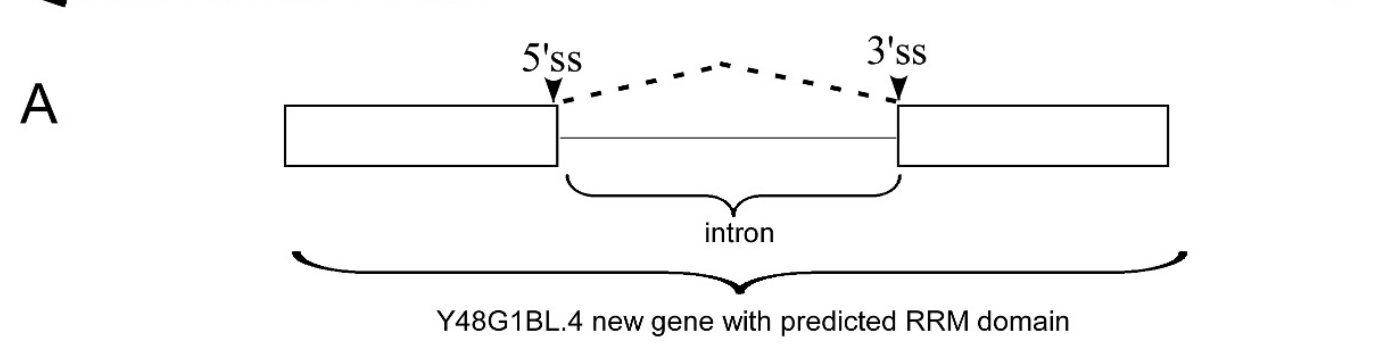

B Y48G1BL.4: 1 TCGGTCGTTTCCCAAATAGAGCATCCGATCGTGATGCCGAGCACTTCTTCTGCGATTATG 60 Harbinger: 345 tcggtcgtttcccaaatagagcatccgatcatgatgccgagcacttcttctgcggatatg 404 Y48G1BL. 4: 61 GAAAGCTGCCGGATGCCATAATGAAGAACGTATTTGGTGTTGGTTGTAAGgtaactattc 120

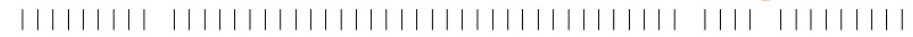
Harbinger: 405 aaaagctgcctgatgccataatgaagaacgtatttggtgttggttataagataactattc 464 Y48G1BL.4: 121 tcatcagaaaccctctagaatgttataatttcagGATTTTCAAGATCAGAGCGATGCTGA 180

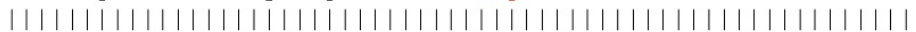
Harbinger: 465 tcatcagaaaccctctagaatgttataatttcaggattttcaagatcagagcgatgctga 524 Y48G1BL. $4: 181$ TGACATCATTTGTGATGAGCAGACAAGAATTTTGCTTGTGGAGAAGCTGAAGAACGCGAA 240

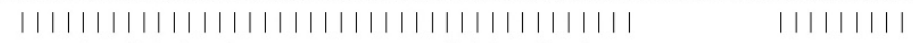
Harbinger: 525 cgacatcatttgtgatgagcagacaagaattttgcttgtgga----------gaacgcgaa 575 Y48G1BL. $4: 241$ GGAATACCCAACAATTAATCACGATTAG 268 1|||||||||||||||||||||||||| $\mid$ Harbinger: 576 ggaatacccaacaattaatcacgattag 603

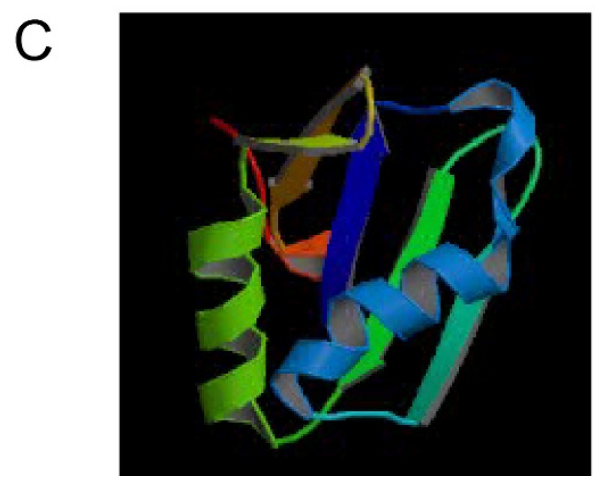

Figure 3

Y48G IBL.4 gene with predicted RRM domain. (A) A schematic illustration of the Y48GIBL.4 gene with predicted exons and an intron, and the position of the Turmoil-I sequence within the genome. (B) Pairwise alignment (using bl2seq) between Turmoil-I and the Y48GIBL.4 DNA sequence. The intron sequence is indicated by lower-case letters and the protein-coding region by upper-case letters. The potential new 5 ' splice site is marked in red and the 5 ' splice site position within the original RRM domain is marked with an arrow. (C) The predicted three-dimensional structure of the RRM domain generated from Y48GIBL. 4. 
One of the Turmoil-1 copies (number 1 in Table 1) contains within it an open reading frame (ORF) with the accession number Y48G1BL.4. It contains two putative exons and an intron (Figure 3), which are similar to the RRM domain. The 5' splice site that corresponds to that in the rsp-2 transcript has been mutated. A novel 5' splice site is most likely located nine nucleotides downstream from the original one. At this site, a point mutation changed an AT into a GT dinucleotide (marked in red in Figure 3). Usage of this 5' splice site maintains the ORF equivalent to that of the RRM domain of rsp- 2 with the exception of the addition of three amino acids. These additional residues should have a negligible effect on the three-dimensional structure of the RRM domain (Figure 3C). This ORF, however, may not be transcriptionally active as its sequence is only found in the UNIPROT database (accession number Q9N3P9), and there is no EST or CDNA supporting evidence. If this is the case, it would be consistent with reports that indicate that all known transduplicates in rice, in spite of their genomic abundance, are pseudogenes [16].

Our analysis indicates that Turmoil-1 of C. elegans has captured two unrelated coding sequences within its ITRs at proximate locations. The presence of a transduplication "hotspot" in this region may be tentatively inferred. This analysis reveals a transduplication of protein-coding regions in $C$. elegans and strengthens the hypothesis that protein domains may be mobilized by transposable elements.

\section{Abbreviations}

RRM: RNA Recognition motif; ITR: inverted terminal repeat; ORF: open reading frame.

\section{Competing interests}

The authors declare that they have no competing interests.

\section{Authors' contributions}

NS and AS did the experiments and analysis. WM interpreted the results. GA and TP supervised the study. NS, AS, WM, TP and GA wrote the paper.

\section{Reviewers' comments \\ Reviewer's report I: Dan Graur, Department of Biology \& Biochemistry University of Houston, Texas, USA \\ A very straightforward report - I have no other comments.}

\section{Reviewer's report 2: William Martin, Institut fuer Botanik III, Heinrich-Heine Universitaet Duesseldorf, Germany} This is an interesting and straightforward paper reporting the presence of transduplication in Caenorhabditis. The report of transduplication in animals would appear to be novel and certainly of sufficient interest to warrant publication. It might be the seed of a larger transduplication avalanche in animals, we'll see. I think the paper is fine for publication with the exception of "open read [ing] frame" in the abstract.

Author's response

Thanks for your comment - the typo was corrected.

\section{Acknowledgements}

We thank Prof. Jerzy Jurka for critical reading of the manuscript. This work was supported by the Israeli Ministry of Science and Technology (MOST) and by grants from the Israel Science Foundation (I449/04 and 40/05), MOP Germany-Israel, GIF, ICA through the Ber-Lehmsdorf Memorial Fund, and DIP and EURASNET. AS is a fellow of the Complexity Science Scholarship program and is supported by a fellowship from the Israeli Ministry of Science.

\section{References}

I. Lev-Maor G, Sorek R, Shomron N, Ast G: The birth of an alternatively spliced exon: $3^{\prime}$ splice-site selection in Alu exons. Science 2003, 300(5623): |288-|29|.

2. Sorek R, Ast G, Graur D: Alu-containing exons are alternatively spliced. Genome Res 2002, I2(7): 1060-1067.

3. Krull M, Brosius J, Schmitz J: Alu-SINE exonization: en route to protein-coding function. Mol Biol Evol 2005, 22(8): I 702-I7II.

4. Krull M, Petrusma M, Makalowski W, Brosius J, Schmitz J: Functional persistence of exonized mammalian-wide interspersed repeat elements (MIRs). Genome Res 2007, I 7(8): I I39- I I 45.

5. Nekrutenko A, Li WH: Transposable elements are found in a large number of human protein-coding genes. Trends Genet 200I, I7(II):619-62I.

6. Sela N, Mersch B, Gal-Mark N, Lev-Maor G, Hotz-Wagenblatt A, Ast $\mathrm{G}$ : Comparative analysis of transposed elements' insertion within human and mouse genomes reveals Alu's unique role in shaping the human transcriptome. Genome Biol 2007, 8(6): R 127.

7. Lorenc A, Makalowski W: Transposable elements and vertebrate protein diversity. Genetica 2003, I I8(2-3): |83-19|.

8. Gotea V, Makalowski W: Do transposable elements really contribute to proteomes? Trends Genet 2006, 22(5):260-267.

9. Chothia C, Gough J, Vogel C, Teichmann SA: Evolution of the protein repertoire. Science 2003, 300(5626): I70I-1703.

10. Pickeral OK, Makalowski W, Boguski MS, Boeke JD: Frequent human genomic DNA transduction driven by LINE-I retrotransposition. Genome Res 2000, I0(4):4I I-4I5.

II. Szak ST, Pickeral OK, Makalowski W, Boguski MS, Landsman D, Boeke JD: Molecular archeology of LI insertions in the human genome. Genome Biol 2002, 3(I0):research0052.

12. Xing J, Wang H, Belancio VP, Cordaux R, Deininger PL, Batzer MA: Emergence of primate genes by retrotransposon-mediated sequence transduction. Proc Natl Acad Sci USA 2006, 103(47): $17608-17613$.

13. Goodier JL, Ostertag EM, Kazazian HH Jr: Transduction of 3'flanking sequences is common in LI retrotransposition. Hum Mol Genet 2000, 9(4):653-657.

14. Hoen DR, Park KC, Elrouby N, Yu Z, Mohabir N, Cowan RK, Bureau TE: Transposon-mediated expansion and diversification of a family of ULP-like genes. Mol Biol Evol 2006, 23(6): I254-I 268.

15. Jiang N, Bao Z, Zhang X, Eddy SR, Wessler SR: Pack-MULE transposable elements mediate gene evolution in plants. Nature 2004, 43 I (7008):569-573.

16. Juretic N, Hoen DR, Huynh ML, Harrison PM, Bureau TE: The evolutionary fate of MULE-mediated duplications of host gene fragments in rice. Genome Res 2005, I5(9): 1292-1297.

17. Zemann A, op de Bekke A, Kiefmann M, Brosius J, Schmitz J: Evolution of small nucleolar RNAs in nematodes. Nucleic Acids Res 2006, 34(9):2676-2685.

18. Schmitz J, Zemann A, Churakov G, Kuhl H, Grutzner F, Reinhardt R, Brosius J: Retroposed SNOfall - a mammalian-wide comparison of platypus snoRNAs. Genome Res 2008, 18(6): 1005-1010. 
19. Kapitonov VV, Jurka J: Molecular paleontology of transposable elements from Arabidopsis thaliana. Genetica 1999, I07(I3):27-37.

20. Jurka J, Kapitonov VV: PIFs meet Tourists and Harbingers: a superfamily reunion. Proc Natl Acad Sci USA 200I, 98(22): $|23| 5-123 \mid 6$.

21. Zhang X, Feschotte C, Zhang Q, Jiang N, Eggleston WB, Wessler SR: $P$ instability factor: an active maize transposon system associated with the amplification of Tourist-like MITEs and a new superfamily of transposases. Proc Natl Acad Sci USA 200I, 98(22): I 2572- I 2577.

22. Jiang N, Bao Z, Zhang X, Hirochika H, Eddy SR, McCouch SR, Wessler SR: An active DNA transposon family in rice. Nature 2003, 421 (6919): 163-167.

23. Kikuchi K, Terauchi K, Wada M, Hirano HY: The plant MITE mPing is mobilized in anther culture. Nature 2003, 42 I(69|9): I67-I70.

24. Kapitonov VV, Jurka J: Harbinger transposons and an ancient HARBII gene derived from a transposase. DNA Cell Biol 2004, 23(5):3||-324.

25. Levy A, Sela N, Ast G: TranspoGene and microTranspoGene: transposed elements influence on the transcriptome of seven vertebrates and invertebrates. Nucleic Acids Res 2008:D47-52.

26. Transpogene Database [http://transpogene.tau.ac.il/]

27. Tatusova TA, Madden TL: BLAST 2 Sequences, a new tool for comparing protein and nucleotide sequences. FEMS Microbiol Lett 1999, 174(2):247-250.

28. Longman D, Johnstone IL, Caceres JF: Functional characterization of SR and SR-related genes in Caenorhabditis elegans. Embo J 2000, I 9(7): |625-1637.

29. Guindon S, Lethiec F, Duroux P, Gascuel O: PHYML Online - a web server for fast maximum likelihood-based phylogenetic inference. Nucleic Acids Res 2005:W557-559.

30. Jones DT, Taylor WR, Thornton JM: The rapid generation of mutation data matrices from protein sequences. Comput Appl Biosci 1992, 8(3):275-282.
Publish with Bio Med Central and every scientist can read your work free of charge

"BioMed Central will be the most significant development for disseminating the results of biomedical research in our lifetime. "

Sir Paul Nurse, Cancer Research UK

Your research papers will be:

- available free of charge to the entire biomedical community

- peer reviewed and published immediately upon acceptance

- cited in PubMed and archived on PubMed Central

- yours - you keep the copyright 